\title{
Quedas em idosos: prevalência e fatores associados
}

\author{
Falls in the elderly: prevalence and associated factors
}

\author{
Caídas en adultos mayores: prevalencia y factores asociados
}

\author{
Alfredo A. Cunha*, Roberto A. Lourenço
}

\begin{abstract}
Resumo
O objetivo do presente estudo foi analisar a prevalência de quedas em idosos e os fatores associados às quedas. Queda foi definida como a mudança de uma posição para outra, de nível inferior, caracterizando uma síndrome geriátrica. Foi observada alta prevalência de quedas em idosos vivendo na comunidade - 30 a $40 \%$ em pessoas acima de 65 anos, sendo que a metade chegou a cair mais de uma vez. A prevalência aumentou com a idade, chegando a 50\% acima dos 80 anos. Na União Europeia, ocorrem, por ano, perto de 40.000 mortes de idosos devido a quedas. Os idosos com mais de 80 anos têm uma taxa de mortalidade associada a quedas seis vezes mais alta do que os idosos entre os 65 e 79 anos, por caírem mais vezes e serem mais frágeis. Nos Estados Unidos, a prevalência foi de 30\%, aumentando para $50 \%$ a partir dos 75 anos. A prevalência de quedas aumenta em subgrupos portadores de deficiência física. No Brasil, diversos autores observaram prevalência entre 30 e $60 \%$. A condição justificou o estudo por sua alta prevalência, suas consequências, sequelas e alto custo. Os fatores associados às quedas em idosos foram identificados através de revisões sistemáticas, tendo sido valorizados dois grupos: intrínsecos e extrínsecos. Os primeiros incluíram fatores hemodinâmicos, uso de medicamentos, doenças neurológicas, doenças neurosensoriais e doenças osteomusculares. Entre os fatores de risco extrínseco citou-se iluminação inadequada, piso escorregadio, objetos ou móveis em locais inadequados e escadas e rampas sem as adequadas adaptações. Concluiu-se que a prevalência de queda em idosos varia de 14\% (estudos populacionais europeus) até $50 \%$ ou mais em idosos acima de 80 anos internados em instituições de longa permanência. A identificação dos fatores de risco dependeu do ambiente, do perfil da população de estudo e do tipo de estudo.
\end{abstract}

Descritores: Acidentes por quedas; Idoso; Epidemiologia; Fatores de risco.

\begin{abstract}
The objective of the present study was to analyze the prevalence of falls in the elderly and factors associated with falls. Fall was defined as a change from one position to another, in a lower level, constituting a geriatric syndrome. It was observed a high prevalence of falls in the elderly living in the community -30 to $40 \%$ in people over 65 years old, half of whom came to fall more than once. The prevalence increased with age, peaking at $50 \%$ above 80 years old. In the European Union, there are, every year, nearly 40,000 elderly deaths due to falls. Senior citizens over 80 years of age have a mortality rate associated with falls six times higher than the elderly between 65 and 79 years of age, since they fall more often and are more frail. In the United States, the prevalence was $30 \%$, increasing to $50 \%$ after 75 years of age. The prevalence of falls increases in subgroups of patients with physical disabilities. In Brazil, several authors have observed a prevalence between 30 and 60\%. This condition, for its high prevalence, consequences, sequelae, and high cost, justified the study. The factors associated with falls in the elderly were identified through systematic reviews, and two groups have been highlighted: intrinsic and extrinsic. The first included hemodynamic factors, use of medications, neurological, neurosensorial and musculoskeletal diseases. Among the extrinsic risk factors cited are inadequate lighting, slippery floors, objects or furniture in inappropriate locations, and stairs and ramps without the appropriate adaptations. It was concluded that the prevalence of falling in the elderly varies from $14 \%$ (European population studies) to $50 \%$ or more in the elderly over 80 years of age interned in long-stay institutions. The identification of risk factors depended on the environment, the study population profile and the type of study.
\end{abstract}

Keywords: Accidental falls; Aged; Epidemiology; Risk factors. 


\section{Resumen}

El objetivo del presente estudio fue analizar la prevalencia de caídas en adultos mayores y los factores asociados a las caídas. La caída fue definida como el cambio de una posición a otra, de nivel inferior, caracterizando un síndrome geriátrico. Se observó alta prevalencia de caídas en adultos mayores viviendo en la comunidad-30\% a 40\% en personas por encima de 65 años, verificándose que la mitad llegó a caer más de una vez. La prevalencia aumentó con la edad, llegando a 50\% por encima de 80 años. En la Unión Europea ocurren, por año, cerca de 40.000 muertes de adultos mayores debido a caídas. Los adultos mayores con más de 80 años tienen una tasa de mortalidad, asociada a caídas, seis veces más alta que la de adultos mayores entre 65 y 79 años, por caer más veces y ser más frágiles. En Estados Unidos la prevalencia fue de 30\%, aumentando para 50\% a partir de 75 años. La prevalencia de caídas aumenta en subgrupos portadores de deficiencia física. En Brasil, varios autores observaron la prevalencia entre 30\% y 60\%. La condición, por la alta prevalencia, por las secuelas y alto costo, justificó el estudio. Los factores asociados a las caídas en adultos mayores fueron identificados a través de revisiones sistemáticas, valorizándose dos grupos: intrínsecos y extrínsecos. Los primeros incluyeron factores hermodinámicos, uso de medicamentos, enfermedades neurológicas, enfermedades neurosensoriales y enfermedades osteomusculares. Entre los factores de riesgo extrínseco se citó: iluminación inadecuada, piso resbaladizo, objetos o muebles en lugares inadecuados, escaleras y rampas sin las adaptaciones adecuadas. Se concluyó que la prevalencia de caídas en adultos mayores varía de $14 \%$ (estudios poblacionales europeos) hasta 50\% o más en adultos mayores por encima de 80 años, internados en instituciones de larga permanencia. La identificación de los factores de riesgo dependió del ambiente, del perfil de la población de estudio y del tipo de estudio.

Palabras clave: Accidentes por caídas; Personas Mayores; Epidemiología; Factores de Riesgo.

\section{Introdução}

Queda no presente estudo significa queda espontânea da própria altura ao solo ou para um nível inferior, caracterizando uma síndrome geriátrica. Inclui-se aqui a queda decorrente do processo de transferência (mudar de posição, como levantar da cama ou de uma cadeira). Ficam excluídas as quedas decorrentes de atividade física com risco de queda: práticas arriscadas como subir em escadas para trocar lâmpadas, práticas esportivas, principalmente aquelas consideradas radicais. Não serão consideradas outras causas de quedas, como as causas externas (acidentes, agressões). ${ }^{1}$

É alta a prevalência e incidência de quedas entre idosos na comunidade. Aproximadamente 35 a $40 \%$ das pessoas acima de 65 anos sofrem pelo menos uma queda por ano. Metade destas chega a sofrer mais de uma queda. A prevalência aumenta com a idade, chegando a $50 \%$ acima dos 80 anos. Tais pacientes sempre necessitam de atendimento, cuja complexidade depende do impacto da queda.'

\section{Prevalência}

Na Europa, o estudo da Rede Europeia para Segurança dos Idosos (European Network for Safety among Elderly) $)^{2}$ chama a atenção para o fato de, na União Europeia, ocorrerem aproximadamente 100.000 casos de lesões fatais decorrentes de quedas ou outro evento por ano, dentre os quais 85.000 são acidentais. Foi observada uma diferença entre os países europeus na ocorrência de morte por acidentes entre os idosos. A Hungria tem a taxa mais elevada, seguida de República Checa, França, Finlândia e Dinamarca. No outro extremo, temos Bulgária, Grécia, Espanha, Reino Unido e Alemanha com as taxas mais baixas.

Na União Europeia ocorrem, por ano, perto de 40.000 mortes de idosos devido a quedas. Os idosos com mais de 80 anos têm uma taxa de mortalidade associada a quedas seis vezes mais alta do que os idosos entre 65 e 79 anos. Isto acontece por caírem mais vezes e por serem mais frágeis. As quedas são a principal causa de lesões nos idosos, sendo que em vários países as quedas representam aproximadamente $75 \%$ de todos os doentes que necessitam de cuidados médicos. A probabilidade da recidiva da queda aumenta com a idade e com a história de quedas, sendo que os mais velhos que caem uma vez têm uma probabilidade duas a três vezes maior de cair outra vez durante o ano seguinte. ${ }^{2}$

Em países menos desenvolvidos, a prevalência de queda entre idosos é alta; por exemplo, na Nigéria foi observada uma taxa de $23 \%$ em estudo com uma amostra de 482 idosos. $^{3}$

Nos Estados Unidos da América do Norte, Finlayson e Peterson relataram que aproximada- 
mente $30 \%$ dos adultos morando na comunidade e com idade a partir de 65 anos sofreram uma queda no último ano. ${ }^{4}$ Considerando-se idosos com 75 anos ou mais, a proporção aumenta para $50 \%$. Se o período fica restrito aos três últimos meses, a proporção é de $16 \%$. A recorrência da queda é comum, tendo sido observada duas ou mais vezes em 409 moradores de comunidade com idade a partir de 65 anos ao longo de 48 semanas.

A prevalência de quedas aumenta entre pessoas com incapacidade física, como, por exemplo, entre os portadores de esclerose múltipla, poliomielite, distrofia muscular e lesões da medula. A idade média dos portadores de esclerose múltipla foi de 40 anos (com desvio padrão de 11 anos), com prevalência de 54\% em 50 pacientes. Em outro estudo foi observada prevalência de $52 \%$ em 1.089 participantes com idade entre 45 e 90 anos. Usando uma amostra da população dos Estados Unidos, foi observada prevalência de 30\% de uma queda e de $64 \%$ de duas ou mais quedas em 354 pacientes com idade entre 55 e 94 anos. $^{4}$

Em estudo com 40 pacientes portadores de sequela de poliomielite, foi observada prevalência de $47,5 \%$ de três ou mais quedas, com um adicional de mais $20 \%$ quando se considerou apenas duas quedas. Outro estudo referiu $84 \%$ de uma queda no último ano entre 172 pacientes. Nos portadores de distrofia muscular foi observada prevalência de $38 \%$ de mais de uma queda nos últimos seis meses. Os pacientes com lesão de medula espinhal apresentaram prevalência de $75 \%$ em 119, com média de idade de 52,2 anos. ${ }^{4}$

No Brasil, diversos autores estudaram a prevalência de quedas em idosos em diferentes ambientes e com características diversas. Em 1996, Rozenfeld ${ }^{5}$ estudou a associação entre quedas e o uso de medicamentos em idosos que deambulam vivendo na comunidade. A autora observou prevalência de 37,4\% entre 634 mulheres.

Chaimowicz e colaboradores estudaram população de idosos vivendo na comunidade e em uso de drogas psicoativas em 1/5 do grupo de estudo. ${ }^{6}$ Observaram prevalência de $16,8 \%$ de quedas e as associaram ao uso dessas drogas. A prevalência de queda entre idosos com 65 anos ou mais foi observada como sendo de $60 \%$ (53/87) por Ferrer e colaboradores em estudo para avaliar a prevalência de fatores ambientais de risco para queda. ${ }^{7}$ Siqueira e colaboradores, em estudo transversal incluindo 4.003 idosos, observaram prevalência de $34,8 \%$, significativamente maior nas mulheres (40,1\%); e entre os que já sofreram queda, $12,1 \%$ tiveram fratura como consequência. ${ }^{8}$ Silva e colaboradores observaram prevalência de queda de $29 \%$ em mulheres com idade acima de 60 anos. $^{9}$ Quando consideraram a osteoporose, a prevalência subiu para 51\%. Nascimento e colaboradores observaram prevalência de 39,1\% de quedas entre idosos, dentre os quais 37,9\% apresentaram alteração do equilíbrio. ${ }^{10}$

\section{Fatores de risco}

Barbosa chama a atenção para a avaliação de quedas em idosos quando diz que a instabilidade com a ocorrência de quedas era uma característica do processo de envelhecimento e constitui desafio na medicina geriátrica." A elevada incidência de fratura reforça esta afirmativa, mas o trauma é apenas uma das sequelas da falta de equilíbrio. Além disso, a história de quedas associou-se com aumento da morbidade e da mortalidade.

Este autor utilizou a classificação dos fatores de risco para quedas em intrínsecos e extrínsecos. ${ }^{11}$ Os primeiros incluíram fatores hemodinâmicos (hipotensão ortostática, arritmia cardíaca e várias outras condições cardíacas), uso de drogas (hipotensores, antiarrítmicos, parkinsonianos, anticonvulsivantes), doenças neurológicas (lesões expansivas intracranianas, hidrocéfalo de pressão normal, doenças cerebrovasculares, neuropatia periférica, doença de Parkinson, estados depressivos), doenças neurossensoriais (alterações da visão, da propriocepção e labirintopatias) e doenças osteomusculares. Entre os fatores de risco extrínseco, citou iluminação inadequada, piso escorregadio, objetos ou móveis em locais inadequados e escadas e rampas sem as adequadas adaptações.

King lista como mais frequentes fraqueza muscular, histórico de quedas, deficit na marcha, deficit de equilíbrio, uso de dispositivo de apoio, deficit visual, artrite, redução das atividades básicas da vida diária, depressão, redução da cognição e idade acima de 80 anos. ${ }^{1}$

Finalmente, o assunto é tão importante que a Sociedade Brasileira de Otologia aproveitou para realizar uma etapa adicional da Campanha Nacional de Prevenção a Quedas de Idosos em 
2008, no Dia Nacional do Idoso (27 de setembro). Além do atendimento inicial ao paciente e coleta de dados, a ação visava continuar o trabalho de aumentar o conhecimento do público em geral sobre os problemas relacionados às quedas na terceira idade. ${ }^{12}$

Vemos, assim, que a classificação dos fatores associados a quedas é complexa e depende de múltiplas possibilidades, tais como o cenário do estudo (comunidade ou instituição de longa permanência), o perfil do paciente e os modelos de estudo.

\section{Discussão}

A complexidade do tema justifica o grande número de estudos recuperados das bases de dados bibliográficos, razão pela qual, no presente estudo, nos limitamos às revisões sistemáticas. Estas podem ser abrangentes, quando abordam o tema como um todo, ou restritas a determinadas situações.

\section{Inglaterra}

Em 2004 o Instituto Nacional de Excelência Clínica (National Institute for Clinical Excellence, NICE), da Inglaterra, no propósito de elaborar uma diretriz para a prevenção de quedas em idosos, utilizou as diretrizes da Sociedade Americana de Geriatria e da Sociedade Britânica de Geriatria, ambas de 2001, para fazer uma revisão de fatores de risco de queda em idosos. A instituição considerou 28 estudos e identificou os seguintes fatores de risco: antecedente de queda, comprometimento da mobilidade, comprometimento da visão, deficiência no equilíbrio, deficiência na marcha, comprometimento do estado mental, dependência funcional, medo de cair, deficit ponderal, depressão, diabetes mellitus, risco ambiental, incontinência urinária, medicação múltipla (polifarmácia), uso de antiarrítmico e de substâncias psicoativas. O número de estudos variou de 2 a 11, incluindo metanálises sobre polifarmácia com 14 estudos, antiarrítmico com 10 estudos e substâncias psicoativas com 11 estudos. ${ }^{13}$

Entretanto, para caracterizar a complexidade do tema, havia discordância importante sobre os fatores assinalados na análise multivariada. A revisão dos fatores de risco termina valorizando os seguintes fatores: antecedente de queda, deficiência na marcha, deficiência no equilíbrio, comprometimento da mobilidade, o medo da queda, comprometimento da visão, comprometimento cognitivo, incontinência urinária e o risco do ambiente doméstico. ${ }^{13}$

O Australia Prince of Wales Medical Research Institute, juntamente com Close e colaboradores, fez extensa revisão sobre a força de evidência de associação de fatores intrínsecos de quedas. ${ }^{14} \mathrm{O}$ estudo foi dividido por domínios em psicossocial e demográfico, mobilidade e equilíbrio, marcha, sensório e motor, condições clínicas e medicação, e a força de associação, classificada em três graus avaliados como evidência forte, média e fraca, conforme a informação dos estudos.

No domínio psicossocial e demográfico, foram classificados como evidência forte a idade avançada, a história de quedas, o uso de apoio para caminhar e limitações nas atividades básicas da vida diária. O gênero feminino, o fato de viver só e a inatividade mereceram grau médio. Neste domínio o consumo de álcool não foi valorizado.

No domínio da mobilidade e do equilíbrio, a evidência foi forte para o comprometimento da marcha e da mobilidade, o comprometimento da capacidade de manter-se de pé, e da capacidade de se transferir (mudar da cama para cadeira ou ficar de pé ou o contrário). A redução da capacidade para deitar ou levantar e o caminhar mais lento teve evidência intermediária e a resposta inadequada a imprevistos recebeu grau fraco. ${ }^{14}$

No domínio dos padrões de marcha todas as variáveis avaliadas foram identificadas como fortemente associadas a quedas, a saber: velocidade reduzida da marcha, redução do ritmo, redução da extensão do passo e aumento do tempo de permanência em cada posição. ${ }^{14}$

No domínio sensório e motor foram avaliadas a visão, a sensibilidade, a força e o tempo de reação. Em relação à visão, a sensibilidade reduzida para o contraste visual, a diminuição da percepção de profundidade e a redução da acuidade visual tiveram grau forte de evidência; a perda do campo visual teve grau médio e o aumento da dependência do campo visual teve grau fraco. Em relação à sensação, a redução da sensação periférica não foi valorizada; a redução da função vestibular teve grau médio e a redução da audição teve grau fraco. Quanto à força, a redução da força muscular teve grau forte, enquanto a potência muscular e a 
resistência muscular tiveram grau fraco. O tempo de reação foi avaliado em relação ao tempo de reação simples e ao tempo de escolha, ambos com grau forte de evidência. ${ }^{14}$

No domínio das condições clínicas receberam grau forte o comprometimento da cognição, o antecedente de acidente vascular cerebral, a doença de Parkinson e problemas no pé. Depressão, artrite e alterações de sinais neurológicos receberam grau médio, enquanto vertigem, hipotensão ortostática receberam grau mínimo e as alterações vestibulares não foram valorizadas. ${ }^{14}$

No domínio das medicações receberam grau máximo o uso de múltiplas medicações, medicação psicoativa, benzodiazepínicos, antidepressivos e antipsicóticos. Os anti-hipertensivos tiveram grau mínimo, enquanto os analgésicos e os anti-inflamatórios não foram valorizados. ${ }^{14}$

\section{Brasil - Ministério da Saúde}

O Ministério da Saúde considera a queda em idosos um importante problema de saúde pública, dadas sua alta incidência e suas consequências lesão, incapacidade, institucionalização e morte..$^{15}$

Estima-se em 30\% a incidência anual entre idosos. Esta taxa aumenta para $40 \%$ entre os idosos com mais de 80 anos e $50 \%$ entre os que vivem em instituições de longa permanência. As mulheres tendem a cair mais que os homens até os 75 anos, mas a partir desta idade, as frequências se igualam. Dos que caem, cerca de $2,5 \%$ requerem hospitalização, e desses, apenas a metade sobreviverá após um ano.

Este mesmo órgão classifica as causas mais comuns como: relacionadas ao ambiente; fraqueza/distúrbio de equilíbrio e marcha; tontura/vertigem; alteração postural/hipotensão ortostática; lesão no sistema nervoso central; síncope e redução da visão. É utilizada a classificação dos fatores de risco em intrínsecos e extrínsecos.

Os intrínsecos decorrem das alterações fisiológicas relacionadas ao avançar da idade, da presença de doenças, de fatores psicológicos e de reações adversas de medicações em uso. Podem ser citados: idosos com mais de 80 anos, sexo feminino, imobilidade, quedas precedentes, equilíbrio diminuído, marcha lenta e com passos curtos, baixa aptidão física, fraqueza muscular de membros inferiores e superiores, alterações cognitivas, doença de Parkinson, polifarmácia e uso de sedativos, hipnóticos e ansiolíticos.

Os fatores extrínsecos estão relacionados ao comportamento e atividade da pessoa e ao meio ambiente. Ambientes inseguros e mal iluminados, mal planejados e mal construídos, com barreiras arquitetônicas, representam os principais fatores de risco para queda.

A influência dos fatores ambientais no risco de queda associa-se ao estado funcional e mobilidade da pessoa idosa. Quanto mais frágil, mais suscetível. Manobras posturais e obstáculos ambientais que não são problemas para pessoas idosas mais saudáveis podem transformar-se em séria ameaça à segurança e à mobilidade daquelas com alteração do equilíbrio e da marcha.

Existem fatores que agravam a ocorrência de lesão na queda e podem ser citados: ausência de reflexos de proteção, densidade mineral óssea reduzida e osteoporose, desnutrição, idade avançada, resistência e rigidez da superfície sobre a qual se cai e dificuldades para levantar após a queda. ${ }^{15}$

\section{Espanha}

Outra importante revisão sistemática sobre fatores de risco de quedas em idosos foi feita na Espanha, país com grande experiência em geriatria, considerando a grande prevalência de idosos naquele país. Esta revisão foi publicada no Brasil e é interessante porque pesquisou trabalhos indexados na LILACS e na SciELO, bases de dados bibliográficos que incluem os periódicos de língua portuguesa, espanhola e francesa, línguas utilizadas na pesquisa. Foram identificadas inicialmente 726 publicações, e 15 estudos foram incluídos na revisão sistemática. ${ }^{16}$

Os fatores foram classificados em seis dimensões: sociodemografia, condição clínica e funcional, estado mental, morbidades, uso de fármacos e atividade física. Não foi possível fazer metanálise devido à heterogeneidade dos estudos. O risco foi aferido por diferentes técnicas de acordo com o estudo: razão de chances, risco relativo e razão de densidade de incidência.

$\mathrm{Na}$ dimensão sociodemográfica, na idade avançada o risco variou de 1,01 a 2,50; o risco para o sexo feminino variou de 1,35 a 2,14. A falta de cônjuge representou risco de 1,59. O antecedente de queda teve risco de 1,90 a 3,35.

Na dimensão condição clínica e funcional, o 
risco de queda quando havia alteração de marcha variou de 1,99 a 4,00; com problemas de mobilidade variou de 1,82 a 5,00; com alteração do equilíbrio, de 1,08 a 3,20; com debilidade muscular, de 1,14 a 3,80; com visão prejudicada, de 1,53 a 2,30; com limitação funcional (atividades básicas da vida diária), de 1,05 a 4,94.

O estado mental, avaliado pela perda cognitiva, teve risco de 2,40 a 5,00, e, quando avaliado pela depressão, de 1,59 a 2,20.

As morbidades estiveram representadas por osteoartrite com risco de 1,32 a 2,70; doença de Parkinson, de 1,90 a 3,58; acidente vascular cerebral, de 1,80 a 13,60; diabetes, de 1,37 a 3,60; incontinência urinária, de 1,37 a 1,70; vertigem/ tonteira, de 1,82 a 2,10; hipotensão ortostática, de 1,65 a 3,30.

O uso de fármacos foi estudado quanto à polimedicação e psicofármacos. O risco com a primeira variou de 1,30 a 4,50 e com a segunda, de 1,60 a 28,30, neste limite com uso de antidepressivos, benzodiazepínicos e fenotiazina. Por último, o idoso muito ativo fisicamente corre o risco de queda de 1,09 a 2,00.

Itália

Em outra revisão, Deandrea e colaboradores, partindo de 4.155 estudos identificados na MEDLINE, analisaram 74 estudos e consideraram 31 fatores de risco, classificados como: sociodemográficos, clínicos, psicológicos, medicamentosos, de mobilidade e sensórios. ${ }^{17}$

No primeiro grupo foram considerados os fatores sociodemográficos: idade, sexo, o fato de morar só, antecedente de queda, deficiência física, deficiência funcional, índice de massa corporal, nível de instrução e o uso de apoio para caminhar.

No segundo grupo foram avaliados os fatores clínicos e psicológicos, sendo incluída a presença de comprometimento cognitivo, depressão, antecedente de acidente vascular cerebral, incontinência urinária, doença reumática, vertigem, hipotensão, diabetes mellitus, comorbidade (acréscimo de mais uma doença), a autopercepção do estado de saúde (mau versus bom), dor, medo de cair e doença de Parkinson.

No terceiro grupo foi abordada a medicação usada e avaliado o uso de uma droga comparado a nenhuma e o uso de sedativos, anti-hipertensivos e antiepiléticos, comparado ao não uso.
No quarto grupo foram estudados a mobilidade e o sensório. A mobilidade foi estudada quanto a problemas na marcha, comparado à sua ausência. O sensório incluiu a visão e a audição, que foram avaliados quando havia comprometimento contra o não comprometimento.

Esta metanálise de fatores de risco encontrou razão de chances (odds ratio - OR) entre dois e três para antecedente de queda, vertigem, doença de Parkinson, medo de queda, problemas de marcha, uso de apoio para caminhar e uso de drogas antiepilépticas. Para a maioria dos fatores a razão de chances esteve abaixo de dois. A razão de chance foi geralmente mais alta nos caidores recorrentes (duas ou mais quedas).

\section{Avaliação do risco de quedas em subgrupos}

\section{Pacientes com comprometimento da visão}

Com esse objetivo, Ray e Wolf fizeram revisão dos fatores de risco para queda em grupo específico, o de pacientes com comprometimento da visão, e concluíram que este grupo deve merecer atenção precoce para o deficit da visão que, corrigido, pode diminuir o risco de quedas. ${ }^{18}$

\section{Risco de fratura}

Outro subgrupo que mereceu atenção especial foi o dos pacientes com risco de fratura. Peeters e colaboradores observaram que determinados fatores de risco para queda estão associados a fraturas, com risco maior do que para a simples queda. ${ }^{19} \mathrm{~A}$ idade foi identificada como importante fator de risco, e cada dez anos de aumento na idade correspondem a um RR (relative risk ou risk ratio) de 1,1 para queda e 1,27 (IC =1,22-1,33) para fratura. Quando se comparou mulheres que entraram na menopausa em idade mais jovem com as que o fizeram em idade mais avançada, estas apresentaram um $\mathrm{RR}=1,26(\mathrm{IC}=$ 1,08-1,47) para fratura.

\section{Uso de medicamentos e quedas}

Os idosos têm alta prevalência de morbidades e é frequente a ocorrência de mais de uma doença, levando ao uso de diversos medicamentos. Isto cria um problema de interação das drogas e 
possíveis efeitos colaterais, um dos quais pode ser a queda. A associação entre medicação e queda já vem sendo estudada há bastante tempo, e recentemente Woolcott e colaboradores fizeram uma revisão sistemática do tema. ${ }^{20}$ As drogas avaliadas foram classificadas em nove grupos: anti-hipertensivos, diuréticos, betabloqueadores, sedativos/hipnóticos, neurolépticos/antipsicóticos, antidepressivos, benzodiazepínicos, narcóticos/analgésicos e drogas anti-inflamatórias não esteroides (NSAIDs). É importante chamar a atenção para o pequeno número de estudos com razão de chances ajustadas para confundidores. Concluiu-se que o uso de diuréticos esteve associado a maior risco de queda na metanálise não ajustada, mas não quando foi ajustada para covariáveis. Os anti-hipertensivos foram associados a maior risco de queda. O uso de drogas psicoativas mostrou aumento do risco de queda, especialmente para sedativos e hipnóticos, antidepressivos e benzodiazepínicos. As substâncias neurolépticas e antipsicóticas estiveram associadas a quedas na análise não ajustada, mas não se mostraram estatisticamente associadas quando ajustadas para confundidores.

\section{Anti-inflamatórios não esteroides}

Hegeman e colaboradores publicaram uma revisão sistemática sobre drogas anti-inflamatórias não esteroides (NSAIDs) e o risco de quedas em pacientes idosos. ${ }^{21}$

Não foram localizados ensaios clínicos randomizados e nos treze artigos analisados houve falta de consistência nos métodos estatísticos e muita variação no relato dos desfechos. A média de idade foi muito elevada, o que dificulta a generalização do estudo. Os resultados da revisão mostram tendência a aumento de risco de queda com o uso dos NSAIDs, embora haja divergência entre os estudos, uma vez que quatro foram significantes e nove não o foram.

\section{Antidepressivos}

Outro grupo de substâncias estudadas por Darowski e colaboradores, ${ }^{22}$ separadamente, foi o dos antidepressivos. Estas substâncias têm sido vistas como fatores contribuintes para queda. Existem muitos dados sobre antidepressivos tricíclicos e inibidores de serotonina, mas poucos da- dos sobre outros antidepressivos. Foram também identificados outros fatores contribuintes para a queda, como sedação, insônia e comprometimento do sono, noctúria, diminuição do equilíbrio e do tempo de reação, hipotensão postural, arritmias cardíacas, distúrbios da condução e distúrbios da marcha. Concluiu-se que há poucos dados para rejeitar a hipótese de que os antidepressivos sejam uma causa importante de queda, embora a magnitude do aumento do risco de queda com um antidepressivo seja provavelmente a mesma do que a de um paciente não tratado.

\section{Estado de saúde}

Outro foco específico é o do estado de saúde dos pacientes. Com este objetivo, Harlein e colaboradore $^{23}$ fizeram revisão sistemática sobre o risco de queda em pacientes com demência. A revisão identificou somente seis artigos em língua inglesa e alemã e estudos do tipo caso-controle e de coorte. Entretanto, a diferença de cenário, o tamanho amostral, o tempo de seguimento e as variáveis consideradas não permitiram metanálise. Foi possível somente identificar oito categorias de fatores de risco: comprometimento motor, comprometimento da visão, tipo e grau de demência, alterações de comportamento, comprometimento funcional, história de queda, uso de neurolépticos e baixa densidade mineral óssea. Concluiu-se que há falta de dados para avaliar o risco de queda em pacientes com comprometimento cognitivo. Outros fatores já bem conhecidos como os acima mostram peculiaridades quando ocorrem em pacientes com comprometimento cognitivo.

\section{Deficiência intelectual}

Outra revisão, esta com foco na deficiência intelectual, foi feita por Willgoss e colaboradores. ${ }^{24}$ A revisão foi pouco extensa, abrangendo o período de janeiro de 2007 a janeiro de 2009, embora tenham identificado 177 artigos para seleção, restando artigos que se prestaram à metanálise. Inicialmente, observaram alta prevalência (57\%) de queda em portadores de deficiência intelectual causando lesões em 50-62\%. Observaram que outros fatores estão envolvidos, como idade, comprometimento da mobilidade, epilepsia e problemas comportamentais. Concluiu-se que o 
problema é multifatorial e que esta abordagem é fundamental para uma avaliação abrangente.

\section{Fraqueza muscular}

Outro grupo de pacientes foi aquele com fraqueza muscular. Willgoss avaliou a contribuição da fraqueza muscular na instabilidade postural do idoso em revisão sistemática. $\mathrm{O}$ autor pesquisou indivíduos com mais de 60 anos, sadios, coortes de moradores de comunidades, moradores de casas geriátricas, frágeis, adultos com comprometimento da mobilidade e da funcionalidade. $\mathrm{O}$ objetivo foi observar estudos de intervenção sobre resistência ou força para aumentar a força muscular. Iniciou-se com 74 estudos, acabando com cinco com medida de força e 24 com desfechos de força e potência muscular. Observou-se que houve aumento da força e da potência muscular em $76 \%$ dos estudos, com melhora do equilíbrio. ${ }^{25}$

Ainda na linha de avaliação do aparelho locomotor, Muir e colaboradores estudaram o impacto do comprometimento do equilíbrio sobre o risco de queda. ${ }^{26}$ Foram avaliados estudos de coorte sobre pacientes com mais de 60 anos com pelo menos um ano de acompanhamento e $80 \%$ de seguimento. Analizou-se separadamente 23 artigos e observou-se aumento do risco de queda quando houve comprometimento do equilíbrio. Concluiu-se que o comprometimento do equilíbrio esteve associado a um aumento moderado do risco de queda em idosos morando em comunidades, recomendando-se o uso de escalas de avaliação do equilíbrio em tais pacientes.

\section{Conclusões}

O evento de queda em idoso é de alta prevalência, variando de 14\% (estudos populacionais) até $50 \%$ ou mais quando considerados idosos com mais de 80 anos em instituições de longa permanência.

Os fatores de risco são diversos e podem ocorrer simultaneamente, uma vez que a queda é um evento multifatorial. No entanto, a identificação dos fatores de risco é complexa e depende do ambiente de estudo, das características dos pacientes e dos modelos de estudo.

Recomenda-se a identificação de fatores de risco para fratura causada por queda. $\mathrm{O}$ instru- mento preditivo a ser usado depende do cenário, da população em estudo e das propriedades diagnósticas necessárias.

\section{Referências}

1. King MB. Falls. In: Halter JB, Ouslander JG, Tinetti ME, Studenski S, High K, Asthana S (editors). Hazzard's Geriatric Medicine and Gerontology. New York: McGraw-Hill; 2009. p. 659-69.

2. European Union [homepage on the internet]. European Network for Safety among Elderly. EUNESE [updated 2006; cited 2014 Nov 24]. Available from: http://ec.europa.eu/health/ph_ projects/2003/action3/docs/2003_3_13_inter_en.pdf

3. Bekibele CO, Gureje O. Fall incidence in a population of elderly persons in Nigeria. Gerontology. 2010;56(3):278-83.

4. Finlayson ML, Peterson EW. Falls, aging. and disability. Phys Med Rehabil Clin N Am. 2010;21(2):357-73.

5. Rozenfeld S, Camacho LAB, Veras RP. Medication as a risk factor for falls in older women in Brazil. Rev Panam Salud Pública. 2003;13(6):369-75.

6. Chaimowicz F, Ferreira TJXM, Miguel DFA. Use of psychoactive drugs and related falls among older people living in a community in Brazil. Rev Saúde Pública. 2000;34(6):631-5.

7. Ferrer MLP, Perracini MR, Ramos LR. Prevalência de fatores ambientais associados a quedas em idosos residentes na comunidade em São Paulo, SP. Rev Bras Fisioter. 2004;8(2):149-54.

8. Siqueira FV, Fachini LA, Piccini RX, Tomasi E, Thumé E, Silveira DS, et al. Prevalência de quedas em idosos e fatores associados. Rev Saúde Publica. 2007;41(5):749-56.

9. Silva RS, Costa-Paiva L, Oshima MM, Morais SS, Pinto-Neto AM. Frequência de quedas e associação com parâmetros estabilométricos de equilíbrio em mulheres na pós-menopausa com e sem osteoporose. Rev Bras Ginecol Obstet. 2009;31(10):496-502.

10. Nascimento BN, Duarte BV, Antonini DG, Borges SM. Risco para quedas em idosos da comunidade: relação entre tendência referida e susceptibilidade para quedas com o uso do teste clínico de interação sensorial e equilíbrio. Rev Soc Bras Clin Méd. 2009;7(2):95-9.

11. Barbosa MT. Como avaliar quedas em idosos? Rev Assoc Med Bras. 2001;47(2):93-4.

12. Ganança FF, Mezzalira R, Cruz OLM. Campanha nacional de prevenção a quedas de idosos dia 27 de setembro: dia de atendimento ao idoso com tontura. Editorial. Rev Bras Otorrinolaringol. 2008;74(2):162. 
13. National Institute for Clinical Excellence (NICE). Clinical practice guideline for the assessment and prevention of falls in older people. London: Royal College of Nursing; 2004.

14. Close JC, Lord SL, Menz HB, Sherrington C. What is the role of falls? Best Pract Res Clin Rheumatol. 2005;19(6):913-35.

15. Ministério da Saúde. Envelhecimento e Saúde da Pessoa Idosa. Cadernos de Atenção Básica n ${ }^{0} 19$. Brasília: Ministério da Saúde; 2006. Cap. 12. Quedas; p. 67-70.

16. Gama ZAS, Gómez-Comesa A. Factores de riesgo de caídas em ancianos: revision sistemática. Rev Saúde Pública. 2008;42(5):946-56.

17. Deandrea S, Lucenteforte E, Bravi F, Foschi R, La Cecchia C, Negri E. Risk factors for falls in community-dwelling older people: a systematic review and meta-analysis. Epidemiology. 2010;21(5):658-68.

18. Ray CT, Wolf SL. Review of intrinsic factors related to fall risk in individual with visual impairments. J Rehabil Res Dev. 2008;45(8):1117-24.

19. Peeters G, van Schoor NM, Lips P. Fall risk: the clinical relevance of falls and how to integrate fall risk with frature risk. Best Pract Res Clin Rheumatol. 2009;23(6):797-804.

20. Woolcot JC, Richardson KJ, Wiens MO, Patel B, Marin

Recebido: 08/03/2013.

Revisado: 26/10/2013.

Aprovado: 09/12/2013.
J, Khan KM, et al. Meta-analysis of the impact of 9 medication classes on falls in elderly persons. Arch Intern Med. 2009;169(21):1952-60.

21. Hegeman J, van den Bemt BJ, Duysens J, van Limbeek J. NSAIDs and the risk of accidental falls in the elderly: a systematic review. Drug Saf. 2009;32(6):489-98.

22. Darowski A, Chambers SA, Chambers DJ. Antidepressants and falls in the elderly. Drugs Aging. 2009;26(5):381-94.

23. Härlein J, Dassen T, Halfens RJ, Heinze C. Fall risk factors in older people with dementia or cognitive impairment: a systematic review. J Adv Nurs. 2009;65(5):922-33.

24. Willgoss TG, Yohannes AM, Mitchell D. Review of risk factors and preventive strategies for fall-related injuries in people with intellectual disabilities. J Clin Nurs. 2010;19(15-16):2100-9.

25. Orr R. Contribution of muscle weakness to postural instability in the elderly. Eur J Phys Rehabil Med. 2010;46(2):183-220.

26. Muir SW, Berg K, Chesworth B, Klar N, Speechley $M$. Quantifying the magnitude of risk for balance impairment on falls in community-dwelling older adults: a systematic review and meta-analysis. J Clin Epidemiol. 2010;63(4):389-406.

\section{Alfredo A. Cunha}

Laboratório de Pesquisa em Envelhecimento Humano GeronLab. Departamento de Medicina Interna. Faculdade de Ciências Médicas. Universidade do Estado do Rio de Janeiro. Rio de Janeiro, RJ, Brasil.

\section{Roberto A. Lourenço}

Departamento de Medicina Interna. Faculdade de Ciências Médicas. Universidade do Estado do Rio de Janeiro. Rio de Janeiro, RJ, Brasil. 